\title{
A Dynamic Variance-Based Triggering Scheme for Distributed Cooperative State Estimation over Wireless Sensor Networks
}

\author{
Hongbo Zhu $(\mathbb{D}$ and Jiabao Ding \\ School of Electrical and Information Engineering, Anhui University of Science and Technology, Huainan 232001, China \\ Correspondence should be addressed to Hongbo Zhu; hbzhu@aust.edu.cn
}

Received 20 July 2020; Revised 11 December 2020; Accepted 14 December 2020; Published 4 January 2021

Academic Editor: Atila Bueno

Copyright (c) 2021 Hongbo Zhu and Jiabao Ding. This is an open access article distributed under the Creative Commons Attribution License, which permits unrestricted use, distribution, and reproduction in any medium, provided the original work is properly cited.

\begin{abstract}
Wireless sensor networks (WSNs) have been spawning many new applications where cooperative state estimation is essential. In this paper, the problem of performing cooperative state estimation for a discrete linear stochastic dynamical system over wireless sensor networks with a limitation on the sampling and communication rate is considered, where distributed sensors cooperatively sense a linear dynamical process and transmit observations each other via a common wireless channel. Firstly, a novel dynamic variance-based triggering scheme (DVTS) is designed to schedule the sampling of each sensor and the transmission of its local measurement. In contrast to the existing static variance-based triggering scheme (SVTS), the newly proposed DVTS can lead to the larger average intertrigger time interval and thus fewer total triggering number with almost approximate estimation accuracy. Second, a new Riccati equation of the prediction variance iteration for each estimator is obtained, which switches dynamically among the modes related to the variance of the previous step and the recently received measurements from other sensors. Furthermore, the stability issue is also mainly investigated. Finally, simulation results show the effectiveness and advantage of the proposed strategy.
\end{abstract}

\section{Introduction}

Wireless sensor networks (WSNs) are composed of a wealth of low-cost tiny devices that integrate heterogeneous sensing and wireless communication with limited processing and power resources [1-3]. They have spawned a wide range of applications, including cooperative target localization and tracking [4, 5], environment and health monitoring [6], control and guidance of unmanned vehicles or mobile robots $[7,8]$, and smart grids [9].

In these applications, the nodes of sensor networks are distributed in the determined areas or deployed randomly in the unknown areas without power supplying to monitor some specific targets and powered by independent power resource such as onboard batteries. Then, how to save power energy of the WSNs to prolong its lifespan is a fundamental issue we have to consider. Obviously, the lifespan of the WSNs is directly related to the average amount of sensing, processing, and communicating of each sensor node. Hence, it is a direct and effective way of improving energy-efficiency of the WSNs to reduce appropriately the average rate of sensing, processing, and communication carried out by each sensor node and also to maintain the acceptable overall estimation performance in the meanwhile [10].

In addition, as the application of large-scale WSNs increases, the cost of communication among the sensor nodes becomes a pivotal factor that we have to pay attention to, in that it is related to the overall performance of control systems. Clearly, it is an effective way of easing the excessive concentration pressure of communication traffic degrading the overall performance of control systems or estimators to reduce the average communication rate of the large-scale WSNs appropriately $[11,12]$.

The abovementioned considerations have led to the great development of sensor triggering or scheduling mechanisms that can make a better tradeoff between the average rate of communication and the overall estimation performance related usually to the estimation error, usually known as controlled communication $[13,14]$. The tradeoff between the average rate of communication and the overall estimation 
performance is explored in [15-18]. In the works of [15, 16], the existence of a critical value of the observations loss rate within bounded estimation error covariance has been proved, which allows tradeoff between energy-efficiency and estimation accuracy.

Event-triggered or data-driven framework is popular one of improving energy-efficiency of the overall sensor networks and ensuring use-efficiency of the communication resource in WSNs $[12,19-22]$. In contrast to the traditional time-driven or time-triggered framework, the data-driven or event-triggered one makes each sensor node communicate or work only when some index data are required to meet the specific constraint on the overall performance of control system or estimator. For example, the WSNs-based state estimation problem with deterministic event-triggering scheme is studied in [18, 23-25], which has a common disadvantage that Gaussian property of innovation process is easily destroyed by intermittent measurement sequences, but is easy to implement. Then, to maintain the Gaussian property of innovation process, the WSNs-based state estimation problem with stochastic event-triggering scheme is studied in [19, 26-30].

In the abovementioned references on the WSNs-based state estimation problem with deterministic event-triggered or data-driven scheduling scheme, an event of observation transmission is usually triggered by some specified conditions on real-time measurement or state, and in the stochastic event-triggered scheduling scheme, observation is usually transmitted to the base node in a random triggering mode or observation transmission is triggered by a random event. As opposed to the eventtriggered scheduling schemes, the variance-triggering framework used herein depends on the prediction variance at the current step, whose resulting variance iteration is deterministic and only related to the available measurements and the estimation variance $[12,31]$. In the works of $[12,31]$, each sensor node is regarded as an independent estimator running a copy of the Kalman filter and transmits its measurement to the base node only when the associated measurement prediction variance of each sensor node exceeds the allowable or tolerable upper bounds, which can be categorized as static variance-based triggering scheme (SVTS) as the thresholds are fixed all the time. Under the SVTS, the sensor transmission event is triggered once the prediction variance exceeds the static thresholds. In the work of [32], a dynamic event-triggered transmission scheme (DETS) is presented for distributed set-membership estimation over wireless sensor networks, which can result in larger interevent time interval in contrast to the static event-triggered transmission scheme (SETS). Under the DETS, each sensor node is also regarded as an independent estimator running a copy of the set-membership filter and transmits its local measurement to its nearby sensors only when the deviation between its current measurement and its last transmitted measurement exceeds an auxiliary offset variable that can be adjusted real time in accordance with the last deviation. In the DETS, the auxiliary offset variable just is adjusted with the last deviation, but not related with the last triggered status of all sensors.

Hence, motivated partially by the SVTS proposed in [12] and the DETS proposed in [32], we expect intuitively that if the threshold could be adjusted dynamically to make the prediction variance not easily surpass the dynamic threshold, the total triggering number would be further decreased than the one by using SVTS. Thus, it is the main motivation of this paper to design and develop an efficient DVTS for distributed cooperative estimation over wireless sensor networks.

In this paper, we consider the remote cooperative state estimation problem for a discrete linear stochastic dynamical system over wireless sensor networks with a limitation on the sampling and communication rate. The dynamical system is observed by a group of spatially distributed or deployed smart sensors with limited power resource, which communicates each other via a common wireless network channel. Each smart sensor can measure the output of the plant, process its measurement, and transmit its processed measurement to other nearby sensors, as shown in Figure 1. The main novelty and contributions of this paper are summarized as follows:

(i) A new DVTS is designed and developed to determine when each smart sensor should sense the plant and transmit its processed measurement to its neighbors. In contrast to the SVTS, an auxiliary dynamic offset variable with initial nonnegative offset is introduced into the threshold, which is analytically proved to be nonnegative all the time, and it is also analytically proved that the intertrigger time interval generated by the DVTS is no less than the one caused by the SVTS. In this sense, the total triggering numbers by the DVTS are no more than the one by the STVS in the same time interval.

(ii) A novel type of Riccati equation, which dynamically switches among the modes depending on the variance of the previous step and the recently received measurements from other nearby sensors, is derived and obtained as a critical element or condition of the DVTS for this remote cooperative estimator.

(iii) The stability properties of the proposed distributed cooperative estimation method are analyzed and studied, and we derive and prove that the estimation error is asymptotically bounded under system collective observability and controllability.

The remainder of this paper continues as follows. In Section 2, the distributed cooperative state estimator with a DVTS is developed for a discrete linear stochastic dynamical system over wireless sensor networks with a limitation on the sampling and communication rate and the corresponding dynamic iteration Riccati equation for the proposed estimator is derived. The stability properties of the proposed distributed cooperative estimator are analyzed and studied in Section 3. Examples and simulations are provided in Section 4 and Section 5 concludes this paper. 


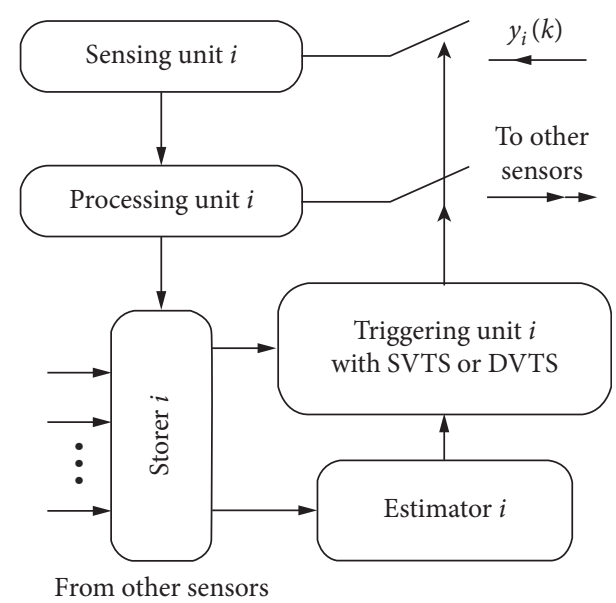

Figure 1: Typical scheduling architecture of a smart sensor with SVTS or DVTS.

1.1. Notations. $\mathbb{Z}_{+}$denotes the set of nonnegative integer numbers, $k \in \mathbb{Z}_{+}$is the sampling time index, $\mathbb{R}$ denotes the set of all real numbers, and $\mathbb{R}^{n}$ is the $n$-dimensional Euclidean space. For a matrix $X \in \mathbb{R}^{n \times n}$ and $i, j=1,2, \ldots, n$, $X \geqslant 0$ denotes that the matrix $X$ is a $n \times n$ semidefinite matrix, $X>0$ denotes that the matrix $X$ is a $n \times n$ definite matrix, $(X)_{i j}$ means to get the element at the $i$-th row and the $j$-th column of the matrix $X$, and $X^{T}$ represents the transposition of $X$. For positive-definite matrices $A$ and $B$, we define $\max \{A, B\} \triangleq\left\{\begin{array}{ll}A & \text { if } A-B \geqslant 0 \\ B & \text { otherwise }\end{array}\right.$, and $\operatorname{diag}(\cdot, \ldots, \cdot)$ denotes the diagonal matrix whose diagonal elements are the entries in $(\cdot) . \mathbf{E}[\cdot]$ denotes the expectation of a random variable, and $\mathbf{E}[\cdot \cdot \cdot]$ is the expectation of a random variable conditioned on another random variable. $\operatorname{Var}[\cdot]$ is the variance of a random variable, and $\operatorname{Var}[\cdot \mid \cdot]$ denotes the variance of a random variable conditioned on another random variable. For functions $f, f_{1}$, and $f_{2}, f_{1} f_{2}(x)$ means the function composition $f_{1}\left(f_{2}(x)\right)$, and $f^{n}(x)=$ $f\left(f^{n-1}(x)\right)$ with $n \in \mathbb{Z}_{+}, f^{0}(x)=x$. $\delta$ function is defined as $\delta_{i j}=1$ if $i=j$; otherwise, $\delta_{i j}=0$. The binary indicator function $1_{F}$ denotes that $1_{F}=1$ if statement $F$ is true, and $1_{F}=0$ if statement $F$ is false.

\section{Variance-Based State Estimator}

In this paper, the overall estimation framework of each smart sensor, as shown in Figure 1, is studied. The model of each system component is provided, and the sensor scheduling strategy and estimation method are presented in this section.

2.1. Process and Sensors. We consider a single linear timeinvariant system with stochastic noises, whose states are observed by a number of $N \in \mathbb{Z}_{+}$distributed sensors

$$
\begin{aligned}
& x(k)=A x(k-1)+\omega(k-1), \\
& y_{i}(k)=C_{i} x(k)+v_{i}(k), \quad i=1,2, \ldots, N .
\end{aligned}
$$

In the above equations, $k$ denotes the discrete sampling time index, $x(k) \in \mathbb{R}^{n}$ is the state vector of the system, $y_{i}(k) \in \mathbb{R}^{m_{i}}$ is the observation taken by sensor $i$, and $n \in \mathbb{Z}_{+}$ and $m_{i} \in \mathbb{Z}_{+}$are the dimensions of the corresponding matrices. The process noise $\omega(k)$ and the observation noise $v_{i}(k)$ are mutually independent processes with $\mathbf{E}\left[\omega(k) v_{i}^{T}(l)\right]=0$ and 0 -mean white Gaussian processes with $\mathbf{E}\left[\omega(k) \omega^{T}(l)\right]=\delta_{k l} Q(Q \geqslant 0)$ and $\mathbf{E}\left[v_{i}(k) v_{j}^{T}(l)\right]=$ $\delta_{k l} \delta_{i j} R_{i}\left(R_{i}>0\right) ; \delta_{k l}$ and $\delta_{i j}$ are the Kronecker delta. In addition, the initial state $x(0)$ is a 0 -mean Gaussian random vector with $\mathbf{E}\left[x(0) x^{T}(0)\right]=\Pi>0$ and is uncorrelated with the process noise $\omega(k)$ and the observation noise $v_{i}(k), \forall k, i$. We also assume that $C_{i}$ is full row rank.

2.2. Estimation Process. As shown in Figure 1, each estimator calculates the estimate of the system state $x(k)$ with the minimum mean-squared estimate error based on the observations received recently from the distributed sensors by using multisensor Kalman filtering. We define

$$
\begin{aligned}
\tilde{y}(k) & \triangleq\left[\left(y_{1}(k)\right)^{T},\left(y_{2}(k)\right)^{T}, \ldots,\left(y_{N}(k)\right)^{T}\right]^{T}, \\
y_{i}(k) & =y_{i}\left(t_{k}^{i}\right) 1_{k-t_{k}^{i} \leq 1}, \quad \text { for } i=1, \ldots, N, \\
\tilde{Y}(k) & \triangleq\{\tilde{y}(1), \tilde{y}(2), \ldots, \tilde{y}(k)\},
\end{aligned}
$$

where $y_{i}\left(t_{k}^{i}\right)$ denotes the received measurement from the smart sensor $i$, which is stored in the buffer, as shown in Figure 1 , and $t_{k}^{i}$ denotes the received time.

$$
\begin{aligned}
\widehat{x}(k \mid k-1) & \triangleq \mathbf{E}[x(k) \mid \tilde{Y}(k-1)], \\
\widehat{x}(k \mid k) & \triangleq \mathbf{E}[x(k) \mid \tilde{Y}(k)], \\
e(k \mid k-1) & \triangleq x(k)-\widehat{x}(k \mid k-1), \\
e(k \mid k) & \triangleq x(k)-\widehat{x}(k \mid k), \\
P(k \mid k-1) & \triangleq \mathbf{E}\left[e(k \mid k-1) e(k \mid k-1)^{T} \mid \tilde{Y}(k-1)\right], \\
P(k \mid k) & \triangleq \mathbf{E}\left[e(k \mid k) e(k \mid k)^{T} \mid \tilde{Y}(k)\right],
\end{aligned}
$$

where $\hat{x}(k \mid k-1)$ and $\hat{x}(k \mid k)$ are called as a priori and a posteriori MMSE estimate, and $P(k \mid k-1), P(k \mid k)$ are the estimation error covariance matrices related to the priori and posteriori MMSE estimate, respectively. At sampling time $k$, the priori estimate state $\widehat{x}(k \mid k-1)$ and corresponding estimation error covariance matrix $P(k \mid k-1)$ are first calculated as follows:

$$
\begin{aligned}
& \widehat{x}(k \mid k-1)=A \widehat{x}(k-1 \mid k-1), \\
& P(k \mid k-1)=A P(k-1 \mid k-1) A^{T}+Q,
\end{aligned}
$$

where the recursion starts from the initial estimation state $\widehat{x}(0 \mid 0)$ and corresponding initial estimation error covariance matrix $P(0 \mid 0)=\Pi$. Let us further define

$$
\begin{aligned}
& \widetilde{C}(k) \triangleq\left(C_{1}^{T} 1_{k-t_{k}^{1} \leq 1}, C_{2}^{T} 1_{k-t_{k}^{2} \leq 1}, \ldots, C_{N}^{T} 1_{k-t_{k}^{N} \leq 1}\right)^{T}, \\
& \widetilde{R}(k) \triangleq \operatorname{diag}\left\{R_{1}+I \sigma_{k-t_{k}^{1} \leq 1}, \ldots, R_{N}+I \sigma_{k-t_{k}^{N} \leq 1}\right\},
\end{aligned}
$$


where $\sigma_{k-t_{k}^{i} \leq 1}=0$ if $k-t_{k}^{i} \leq 1$ is true, and $\sigma_{k-t_{k}^{i} \leq 1}=+\infty$, otherwise, for $i=1,2, \ldots, N$, and the matrix $I$ is the identity matrix with appropriate dimension. At the sampling time $k$, each estimator calculates $\hat{x}(k \mid k)$ and $P(k \mid k)$ as follows:

$$
\begin{aligned}
P(k \mid k) & =\left(P(k \mid k-1)^{-1}+\sum_{i=1}^{N} C_{i}^{T} R_{i}^{-1} C_{i} 1_{k-t_{k}^{i} \leq 1}\right)^{-1} \\
K(k) & =P(k \mid k) \widetilde{C}^{T}(k) \widetilde{R}^{\dagger}(k) \\
\hat{x}(k \mid k) & =\hat{x}(k \mid k-1)+K(k)(\tilde{y}(k)-\tilde{C}(k) \hat{x}(k \mid k-1))
\end{aligned}
$$

where $\dagger$ denotes the Moore-Penrose pseudoinverse.

Remark 1. If the statements $\left(k-t_{k}^{i} \leq 1, i=1, \ldots, N\right)$ are always true for all time $k$, the recursion (5)-(10) is the wellknown standard Kalman filter for the processes (1) and (2), which keeps track of the Gaussian conditional probability distribution of the system state $x(k)$ that is conditioned on all observations from the distributed sensors up to time $k$. To distinguish this well-known standard Kalman filter from our proposed variance-triggered filter as well as the other eventtriggered or data-driven filters, we call it as the full communication Kalman filter [12]. For the full communication Kalman filter, the state prediction estimation error variance $\operatorname{Var}\left[x(k) \mid Y(k-1)=\left\{y_{1}(k-1), y_{2}(k-1), \ldots\right.\right.$,

$\left.\left.y_{N}(k-1)\right\}\right]$ converges to $\bar{P}>0$ of the unique positive semidefinite solution to the discrete algebraic Riccati equation (DARE) for the processes (1) and (2):

$$
\begin{aligned}
& \bar{P}=A \bar{P} A^{T}+Q-A \bar{P} C^{T}\left(C \bar{P} C^{T}+R\right)^{-1} C \bar{P} A^{T}, \\
& C \triangleq\left(C_{1}^{T}, C_{2}^{T}, \ldots, C_{N}^{T}\right)^{T}, \\
& R \triangleq \operatorname{diag}\left\{R_{1}, R_{2}, \ldots, R_{N}\right\} .
\end{aligned}
$$

For the notational simplicity and convenience, we use $\bar{P}=\operatorname{DARE}(A, C, Q, R)$ instead of equation (11).

2.3. Dynamic Variance-Based Triggering Scheme. It is noteworthy that a process or plant is observed by many distributed smart sensors with wireless communication unit, such as target tracking and environment monitoring, where sensing data redundancy should be considered besides power and bandwidth resources due to various design and implementation considerations. Therefore, from the perspective of resource conservation and full utilization, it is inappropriate and improvident to make each sensor observe the plant and transmit its observation to its neighbors all the time. So, it is indispensable to develop an effective triggering scheme to reduce the average rate of sensor's observations and transmissions by taking full advantage of the redundancy of all recently received broadcast data from other sensors. Motivated by a static variance-based triggering scheme proposed in [12] and a dynamic event-triggered transmission scheme proposed in [32], a new dynamic variance-based triggering scheme is presented in this section.

With consideration of notational simplicity and convenience, we use $P_{k} \triangleq P(k \mid k-1)$ to denote the state prediction variance. We know that the state prediction variance $P_{k}$ reflects the uncertainty of the prediction state $\hat{x}(k \mid k-1)$ with all available observations $\tilde{Y}(k-1)$ up to the previous step time $k-1$. Hence, the estimator can schedule the smart sensor to observe the plant and transmit its observation according to the difference between the prediction variance of the dynamically variance-triggered Kalman filter and the steady-state prediction variance of the full communication Kalman filter $\quad P_{k}-\bar{P}=\operatorname{Var}[x(k) \mid \widetilde{Y}(k-1)]-$ $\lim _{k \rightarrow+\infty} \operatorname{Var}[x(k) \mid Y(k-1)]$.

Therefore, we define the triggering variable $\gamma_{k}^{i}$ and the dynamic auxiliary variable $\varepsilon_{k}^{i}$ as follows:

$$
\begin{gathered}
\gamma_{k}^{i}= \begin{cases}1, & \text { if } \varepsilon_{k}^{i}>\delta_{k}^{i}, \\
0, & \text { otherwise, }\end{cases} \\
\varepsilon_{k}^{i}=C_{i}\left(P_{k}-\bar{P}\right) C_{i}^{T}-\sigma_{i}, \\
\delta_{k+1}^{i}=\rho_{k+1}^{i} \delta_{k}^{i}-\left(\rho_{k}^{i}-\rho_{k+1}^{i}\right) \varepsilon_{k}^{i}, \\
0<\rho_{k}^{i}=\frac{1+\sum_{l=k-L}^{k-1} \gamma_{l}^{i}}{L} \leq 1,
\end{gathered}
$$

where $\delta_{k}^{i}$ denotes the auxiliary offset variable related to the recent triggering frequency $\rho_{k}^{i}$ of the sensor $i$ with $\delta_{0}^{i}$ representing the initial offset of the auxiliary system, and $L \in \mathbb{Z}_{+}$ represents the time-window.

Remark 2. Compared with the static variance-based triggering scheme proposed in [12],

$$
\gamma_{k}^{i}= \begin{cases}1, & \text { if } C_{i}\left(P_{k}-\bar{P}\right) C_{i}^{T}-\sigma_{i}>0 \\ 0, & \text { otherwise }\end{cases}
$$

where the design parameter $\sigma_{i}$ is constant, and the introductions of $\delta_{k}^{i}$ and $\rho_{k}^{i}$ are critical ingredients of the dynamic variance-based triggering scheme (12)-(15), which is partially motivated by the dynamic event-triggered transmission scheme proposed in [32] for distributed setmembership estimation, whereas the dynamic event-based triggering scheme in [32] required all smart sensors to observe and measure the plant or process all the time, which does not take advantage of the redundancy of sampling data from other sensors. In contrast, the dynamic variance-based triggering mechanism (12)-(15) is based on the recently received sampling data from other sensors and the prediction variance to schedule each sensor's observation and transmission. In the meanwhile, the dynamic auxiliary variable $\varepsilon_{i}(k)$ is delicately established such that the dynamic variance-based triggering scheme (12)-(15) can dynamically regulate the ratio of triggered sensors to all sensors, and the auxiliary offset variable $\delta_{k}^{i}$ is also delicately constructed such that the one can dynamically regulate the intertrigger time interval. In this sense, the value of $\delta_{k}^{i}$ and $\rho_{k}^{i}$ could be 
adaptively adjusted in real time according to the amount of recently received observations from other sensors and its latest triggered status.

Lemma 1. For given initial offset $\delta_{0}^{i} \geq 0$ and time-window $L \in \mathbb{Z}_{+}$in the dynamic variance-based triggering mechanism (12)-(15), the auxiliary offset variable $0 \leq \delta_{k}^{i} \leq \delta_{0}^{i}$ always holds for all $k \in \mathbb{Z}_{+}$.

Proof. Under the dynamic variance-based triggering scheme (12)-(15), there are two cases including $\gamma_{k}^{i}=1$ and $\gamma_{k}^{i}=0$.

For the first case $\gamma_{k}^{i}=0$, considering the worse situation $\gamma_{l}^{i}=0$ for all $1 \leq l \leq k$, we have that

$$
\varepsilon_{k}^{i} \leq \delta_{k}^{i} .
$$

Then, (15) indicates that

$$
\begin{aligned}
\rho_{k}^{i}-\rho_{k+1}^{i} & \leq \frac{1}{L}, \\
\rho_{k+1}^{i} & \geq \frac{1}{L} .
\end{aligned}
$$

Combining (18) and (19) means that

$$
\rho_{k+1}^{i}-\left(\rho_{k}^{i}-\rho_{k+1}^{i}\right)=2 \rho_{k+1}^{i}-\rho_{k}^{i} \geq \frac{1}{L}-\frac{1}{L} \geq 0 .
$$

According to (14), combining (17) and (20) leads to that

$$
\begin{aligned}
\delta_{k+1}^{i} & =\rho_{k+1}^{i} \delta_{k}^{i}-\left(\rho_{k}^{i}-\rho_{k+1}^{i}\right) \varepsilon_{k}^{i}, \\
& \geq\left(2 \rho_{k+1}^{i}-\rho_{k}^{i}\right) \delta_{k}^{i} \geq \cdots \geq \prod_{l=0}^{k}\left(2 \rho_{l+1}^{i}-\rho_{l}^{i}\right) \delta_{0}^{i} \geq 0 .
\end{aligned}
$$

For the another case, $\gamma_{k}^{i}=1$ and from (22), that means $\varepsilon_{k}^{i}>\delta_{k}^{i} \geq 0$. Then, we can have that $\rho_{k}^{i}-\rho_{k+1}^{i} \leq 0$ from (15). Thus, (14) indicates that

$$
\delta_{k+1}^{i}=\rho_{k+1}^{i} \delta_{k}^{i}-\left(\rho_{k}^{i}-\rho_{k+1}^{i}\right) \varepsilon_{k}^{i} \geq \rho_{k+1}^{i} \delta_{k}^{i} \geq 0 .
$$

Thus, combining two cases, we can conclude that the auxiliary offset variable $\delta_{k}^{i} \geq 0$ always holds for all $k \in \mathbb{Z}_{+}$.

Lemma 2. Under the dynamic variance-based triggering scheme (12)-(15), if $\gamma_{M}^{i}=1, \gamma_{N}^{i}=1$, and $\gamma_{k}^{i}=0$ with $M<k<N, M, N, k \in \mathbb{Z}_{+}$, then (i) the auxiliary offset variable $\delta_{k}^{i} \geq \delta_{k+1}^{i}$ always holds for all $\varepsilon_{k}^{i} \geq-\delta_{k}^{i}$, and (ii) the auxiliary offset variable $\rho_{k}^{i} \delta_{k}^{i} \leq \delta_{k+1}^{i} \leq \rho_{k+1}^{i} \delta_{k}^{i}+\left(\sigma_{i} / L\right)$ always holds for all $\varepsilon_{k}^{i}<-\delta_{k}^{i}$.

Proof. From (12) and (15), we have that

$$
\varepsilon_{k}^{i} \leq \delta_{k}^{i}, \rho_{k}^{i}-\rho_{k+1}^{i} \geq 0 .
$$

Thus, for $0 \leq \varepsilon_{k}^{i} \leq \delta_{k}^{i}$, (14) and (24) indicate that

$$
\delta_{k+1}^{i}=\rho_{k+1}^{i} \delta_{k}^{i}-\left(\rho_{k}^{i}-\rho_{k+1}^{i}\right) \varepsilon_{k}^{i} \leq \rho_{k+1}^{i} \delta_{k}^{i} \leq \delta_{k}^{i} .
$$

Then, for $-\delta_{k}^{i} \leq \varepsilon_{k}^{i}<0$, (14) and (24) yield that

$$
\begin{aligned}
\delta_{k+1}^{i} & =\rho_{k+1}^{i} \delta_{k}^{i}-\left(\rho_{k}^{i}-\rho_{k+1}^{i}\right) \varepsilon_{k}^{i} \\
& \leq \rho_{k+1}^{i} \delta_{k}^{i}+\left(\rho_{k}^{i}-\rho_{k+1}^{i}\right) \delta_{k}^{i} \leq \rho_{k}^{i} \delta_{k}^{i} \leq \delta_{k}^{i} .
\end{aligned}
$$

Combining (25) and (26) gives $\delta_{k}^{i} \geq \delta_{k+1}^{i}$ for $\varepsilon_{k}^{i} \geq-\delta_{k}^{i}$. Finally, for $\varepsilon_{k}^{i}<-\delta_{k}^{i}$, combining (14) and (24) yields

$$
\delta_{k+1}^{i}=\rho_{k+1}^{i} \delta_{k}^{i}-\left(\rho_{k}^{i}-\rho_{k+1}^{i}\right) \varepsilon_{k}^{i} \geq \rho_{k}^{i} \delta_{k}^{i} .
$$

In addition, $C_{i}\left(P_{l}-\bar{P}\right) C_{i}^{T} \geq 0$ always holds for all $l \in \mathbb{Z}_{+}$; it leads to $\varepsilon_{k}^{i} \geq-\sigma_{i}$. Thus, we have that

$$
\delta_{k+1}^{i}=\rho_{k+1}^{i} \delta_{k}^{i}-\left(\rho_{k}^{i}-\rho_{k+1}^{i}\right) \varepsilon_{k}^{i} \leq \rho_{k+1}^{i} \delta_{k}^{i}+\left(\frac{\sigma_{i}}{L}\right) .
$$

Combining (27) and (28) yields $\rho_{k}^{i} \delta_{k}^{i} \leq \delta_{k+1}^{i} \leq \rho_{k+1}^{i} \delta_{k}^{i}+$ $\left(\sigma_{i} / L\right)$ for $\varepsilon_{k}^{i}<-\delta_{k}^{i}$.

Lemma 1 shows that the auxiliary offset variable $\delta_{k}^{i}$ is always nonnegative with initial offset $\delta_{0}^{i} \geq 0$ for all $k \in \mathbb{Z}_{+}$, which makes the triggering condition in the dynamic variance-based triggering scheme (12)-(15) more stringent than the one in the static variance-based triggering scheme (16), and it is shown in Lemma 2 that the triggering condition in (12)-(15) can be adaptively slightly relaxed as the recent triggered frequency decreases, which makes the overall estimation accuracy be guaranteed to some extent.

Lemma 3. Under the dynamic variance-based triggering scheme (12)-(15), let $\gamma_{M}^{i}=1, \gamma_{N_{d}}^{i}=1$, and $\gamma_{k}^{i}=0$ for $M<k<N_{d}, M, N_{d}, k \in \mathbb{Z}_{+}$with initial offset $\delta_{0}^{i} \geq 0$, and under the static variance-based triggering scheme (16), let $\gamma_{M}^{i}=1, \gamma_{N_{s}}^{i}=1$, and $\gamma_{k}^{i}=0$ for $M<k<N s, M, N_{s}, k \in \mathbb{Z}_{+}$. Then, we have that $N_{s} \leq N_{d}$.

Proof. Supposing $N_{s}>N_{d}$, from (16), we can have that

$$
C_{i}\left(P_{N_{d}}-\bar{P}\right) C_{i}^{T}-\sigma_{i}=\varepsilon_{N d}^{i} \leq 0 .
$$

In addition, from (12), we also have that

$$
\varepsilon_{N_{d}}^{i}>\delta_{N_{d}}^{i} .
$$

According to Lemma 1, we know that $\delta_{k}^{i} \geq 0$ always hold for all $k \in \mathbb{Z}_{+}$because of $\delta_{0}^{i} \geq 0$. Then, from (30), we have that $\varepsilon_{N_{d}}^{i}>\delta_{N_{d}}^{i} \geq 0$, but from (29), we also have that $\varepsilon_{N_{d}}^{i} \leq 0$, which leads to contradiction. Thus, by contradiction, we have that $N_{s} \leq N_{d}$, the proof has been completed.

Lemma 3 shows that the intertrigger time interval generated by applying the dynamic variance-based triggering scheme (12)-(15) is no less than the one by using the static variance-based triggering scheme (16), which indicates that the total triggering number and totally released data packets resulted by the dynamic variance-based triggering scheme (12)-(15) will be no more than the ones resulted by the static variance-based triggering scheme (16).

2.4. Dynamic Switching Riccati Equation. It is easy to obtain that the update iteration equation of the state prediction variance $P_{k}$ for $i$ th smart sensor can be written as follows: 


$$
P_{k+1}=A\left(P_{k}^{-1}+\sum_{i=1}^{N} C_{i}^{T} R_{i}^{-1} C_{i} 1_{k-t_{k}^{i} \leq 1}\right)^{-1} A^{T}+Q .
$$

It is easy to see from equation (31) that the state prediction variance $P_{k+1}$ is the function of the state prediction variance $P_{k}$ and the recently received measurements from other smart sensors and itself. For notational simplicity and convenience, let us define $P_{k+1} \triangleq H\left(P_{k}, 1_{k-t_{k}^{i} \leq 1}\right)$, where $H(\cdot, \cdot)$ denotes the map from $\left(P_{k}, 1_{k-t_{k}^{i} \leq 1}\right)$ to $P_{k+1}$. Since the binary indicator function $1_{k-t^{i} \leq 1}$ related to the recently received measurements equals to either 1 or 0 , the update iteration Riccati equation (31) varies dynamically with the status of each binary indicator function.

As a consequence, the linear system (1) and (2) with the dynamic variance-based triggering scheme (12)-(15) can be regarded as a dynamic switching system with modes depending on the binary indicator function $1_{k-t_{k}^{i} \leq 1}$ related to the most recently received measurements from other smart sensors and itself. In other words, the system could dynamically switch among the modes as the binary indicator function $1_{k-t_{k}^{i} \leq 1}$, describing the status of the recently received measurements from other smart sensors and itself.

Among these modes, there exist two extreme cases. The first extreme case is that all binary indicator functions from $1_{k-t_{k}^{1} \leq 1}$ to $1_{k-t_{k}^{N} \leq 1}$ equal to 0 , which means that the sensor has not received any measurements in the latest sampling period from any nearby smart sensors including itself. For this case, from (31), we have that

$$
P_{k+1}=A P_{k} A^{T}+Q \triangleq H_{0}\left(P_{k}\right) .
$$

The second extreme case is that all binary indicator functions from $1_{k-t_{k}^{1} \leq 1}$ to $1_{k-t_{k}^{N} \leq 1}$ equal to 1 , which means that the sensor has received all measurements in the latest sampling period from all nearby smart sensors including itself. For this case, from (31), we have that

$$
P_{k+1}=A\left(P_{k}^{-1}+\sum_{i=1}^{N} C_{i}^{T} R_{i}^{-1} C_{i}\right)^{-1} A^{T}+Q \triangleq H_{1}\left(P_{k}\right) .
$$

To proceed with stability analysis in next section, we present the following theorem.

Theorem 1. If $R_{i}>0$ for $i=1, \ldots, N$ and $P_{0}>0$, then $H_{1}\left(P_{k}\right) \prec P_{k+1}<H_{0}\left(P_{k}\right)$ holds for all $k \in \mathbb{N}$.

Proof. Because of $R_{i} \succ 0$ for $i=1, \ldots, N$, we have that

$$
C_{i}^{T} R_{i}^{-1} C_{i}=\left(\sqrt{R_{i}^{-1}} C_{i}\right)^{T}\left(\sqrt{R_{i}^{-1}} C_{i}\right) \geqslant 0 .
$$

Then, combining the binary indicator function, $1_{k-t_{k}^{i} \leq 1}$, and (34) yields

$$
0 \prec \sum_{i=1}^{N} C_{i}^{T} R_{i}^{-1} C_{i} 1_{k-t_{k}^{i} \leq 1} \prec \sum_{i=1}^{N} C_{i}^{T} R_{i}^{-1} C_{i},
$$

which leads to

$$
\left(P_{k}^{-1}+\sum_{i=1}^{N} C_{i}^{T} R_{i}^{-1} C_{i}\right)^{-1} \prec\left(P_{k}^{-1}+\sum_{i=1}^{N} C_{i}^{T} R_{i}^{-1} C_{i} 1_{k-t_{k}^{i} \leq 1}\right)^{-1} \prec P_{k} .
$$

Thus, from (36), we have that

$$
\begin{aligned}
& A\left(P_{k}^{-1}+\sum_{i=1}^{N} C_{i}^{T} R_{i}^{-1} C_{i}\right)^{-1} A^{T}+Q<A\left(P_{k}^{-1}+\right. \\
& \left.\sum_{i=1}^{N} C_{i}^{T} R_{i}^{-1} C_{i} 1_{k-t_{k}^{i} \leq 1}\right)^{-1} A^{T}+Q<A P_{k} A^{T}+Q .
\end{aligned}
$$

According to (32) and (33), we have the result that $H_{1}\left(P_{k}\right) \prec P_{k+1} \prec H_{0}\left(P_{k}\right)$ holds for all $k \in \mathbb{N}$.

\section{Stability Analysis}

In this section, the stability properties of the estimator with the dynamic variance-based triggering scheme are investigated and analyzed.

Let us define

$$
h(X) \triangleq A X A^{T}+Q .
$$

The sequence $G_{k}$ is constructed as follows: $G_{0}=P_{0 \mid 0}$ and $G_{k+1}=h\left(G_{k}\right)$.

Lemma 4. Assuming that $(A, \sqrt{Q})$ is controllable, a sufficient and necessary condition for the sequence $G_{k}$ to converge is that $\rho(A)<1$ or $A$ is stable, where $\rho(A)$ is the spectrum of $A$, and the sequence $G_{k}$ diverges if and only if $\rho(A) \geq 1$ or $A$ is unstable.

Proof. Assume that $A$ is stable or $\rho(A)<1$, and $X=h(X)$ has a unique positive-definite solution $\bar{G}=h(\bar{G})=\sum_{k=0}^{\infty}$ $A^{k} Q\left(A^{T}\right)^{k}$ since $(A, \sqrt{Q})$ is controllable, then

$$
\begin{aligned}
\lim _{k \longrightarrow+\infty} G_{k} & =\lim _{k \longrightarrow+\infty}\left(A^{k+1} G_{1}\left(A^{T}\right)^{k+1}+\sum_{j=0}^{k} A^{j} Q\left(A^{T}\right)^{j}\right) \\
& =\lim _{k \longrightarrow+\infty} \sum_{j=0}^{k} A^{j} Q\left(A^{T}\right)^{j}=\bar{G} \geqslant Q,
\end{aligned}
$$

hence that the sequence $G_{k}$ converges if $\rho(A)<1$ or $A$ is stable has been proved. Moreover, if the sequence $G_{k}$ converges, because of

$$
\lim _{k \longrightarrow+\infty} G_{k}=\lim _{k \longrightarrow+\infty}\left(A^{k+1} G_{1}\left(A^{T}\right)^{k+1}+\sum_{j=0}^{k} A^{j} Q\left(A^{T}\right)^{j}\right),
$$

the right-hand side series denoted by $\bar{G}$ of the above equation converges. It is easy to notice that $\bar{G}$ is the solution to $X=h(X)$. In addition, since the property of controllability and $(A, \sqrt{Q})$ is controllable, $\bar{G}$ is the full-rank and positive-definite matrix. The fact that $\bar{G}=h(\bar{G})$ and $\bar{G}>0$ leads to that the matrix $A$ is stable or $\rho(A)<1$. Hence, it is 
proved that the sequence $G_{k}$ converges if and only if $\rho(A)<1$ or $A$ is stable. Furthermore, $\rho(A) \geq 1$ or that $\mathrm{A}$ is unstable means that the sequence $G_{k}$ does not converge, or means that the sequence $G_{k}$ is unbounded because it is monotonically increasing. Consequently, it has been proved that the sequence $G_{k}$ diverges if and only if $\rho(A) \geq 1$ or $\mathrm{A}$ is unstable.

Lemma 5 (see $[12,33]$ ). Assuming that all binary indicator functions from $1_{k-t_{k} \leq 1}$ to $1_{k-t_{k}^{N} \leq 1}$ equal to 1 for all time $k \in \mathbb{Z}_{+}$, the dynamic switching Riccati equation (31) becomes the Riccati equation of the standard Kalman filter. Then, the prediction variance sequence $\left\{P_{k}\right\}$ converges to $\bar{P} \geqslant Q$ of the unique positive semidefinite solution to the discrete algebraic Riccati equation (DARE), which is given by (11).

Theorem 2. Assume that $(A, \sqrt{Q})$ is controllable, then there exists a unique positive definite satisfying $\bar{P}=H_{1}(\bar{P})$ and a unique positive definite satisfying $\bar{G}=H_{0}(\bar{G})=h(\bar{G})$ such that

$$
\begin{aligned}
& \limsup _{k \longrightarrow \infty} P_{k} \prec \bar{G} \\
& \liminf _{k \longrightarrow \infty} P_{k} \geqslant \bar{P} .
\end{aligned}
$$

If $A$ is stable or $\rho(A)<1$, then $\bar{G}=A \bar{G} A^{T}+Q$, and if $A$ is unstable, then $\bar{G}=\lim _{k \longrightarrow+\infty}\left(A^{k+1} G_{1}\left(A^{T}\right)^{k+1}+\sum_{j=0}^{k} A^{j} Q\right.$ $\left.\left(A^{T}\right)^{j}\right)=+\infty$.

Proof. From Theorem 1, we have that, for all $k \in \mathbb{Z}_{+}$,

$$
H_{1}\left(P_{k-1}\right) \prec P_{k} \prec H_{0}\left(P_{k-1}\right) \text {, }
$$

and when $k \longrightarrow+\infty$, then we have

$$
\begin{aligned}
& \lim _{k \longrightarrow+\infty} H_{1}\left(P_{k-1}\right) \prec \liminf _{k \longrightarrow \infty} P_{k} \prec \lim _{k \longrightarrow+\infty} H_{0}\left(P_{k-1}\right), \\
& \lim _{k \longrightarrow+\infty} H_{1}\left(P_{k-1}\right) \prec \limsup _{k \rightarrow \infty} P_{k} \prec \lim _{k \longrightarrow+\infty} H_{0}\left(P_{k-1}\right) .
\end{aligned}
$$

According to Lemmas 4 and 5 , when $A$ is stable or $\rho(A)<1$, we have

$$
\begin{aligned}
& \bar{P}<\liminf _{k \rightarrow \infty} P_{k}<\bar{G}=A \bar{G} A^{T}+Q, \\
& \bar{P}<\limsup _{k \rightarrow \infty} P_{k}<\bar{G}=A \bar{G} A^{T}+Q,
\end{aligned}
$$

and when $A$ is unstable, we have

$$
\begin{aligned}
& \bar{P} \prec \liminf _{k \longrightarrow \infty} P_{k} \prec \bar{G}, \\
& \bar{P} \prec \limsup _{k \rightarrow \infty} P_{k} \prec \bar{G},
\end{aligned}
$$

where $\bar{G}=\lim _{k \longrightarrow+\infty}\left(A^{k+1} G_{1}\left(A^{T}\right)^{k+1}+\sum_{j=0}^{k} A^{j} Q\left(A^{T}\right)^{j}\right)=$ $+\infty$. This completes the proof.

Theorem 3. Assume that $(A, \sqrt{Q})$ is controllable and $1_{k-t_{k}^{i} \leq 1}=\gamma_{k}^{i}$ (meaning assume that there is no packet loss and time-delay in wireless communications), then there exists a positive-definite matrix $S$ such that the prediction variance $\bar{P}<P_{k}<S$ holds in (31) together with (12)-(15) for all $k \in \mathbb{Z}_{+}$.
Proof. Without loss of generality, for smart sensor $i$ we can assume that there exists a sequence of positive integers $\left\{m_{j} \mid m_{j} \leq m_{j+1}, j \in \mathbb{Z}_{+}\right\}$for such that $\gamma_{k}^{i}=0$ for all $k \neq m_{j}$ and $\gamma_{m_{j}}^{i}=1$ holds. Then, for all $k \neq m_{j}$, we have that

$$
\begin{gathered}
C_{i}\left(P_{k}-\bar{P}\right) C_{i}^{T}-\sigma_{i} \leq \delta_{k}^{i}, \\
C_{i}\left(P_{m_{j}}-\bar{P}\right) C_{i}^{T}-\sigma_{i}>\delta_{m_{j}}^{i} .
\end{gathered}
$$

In addition, from Lemmas 1 and 2, combining (25), (26), and (28) yields that

$$
\delta_{k}^{i} \leq \delta_{0}^{i}+\frac{1}{L} \sigma_{i}
$$

which holds for all $k \in \mathbb{Z}_{+}$. Combining (46) and (48) leads to

$$
C_{i}\left(P_{k}-\bar{P}\right) C_{i}^{T}-\sigma_{i} \leq \delta_{k}^{i} \leq \delta_{0}^{i}+\frac{1}{L} \sigma_{i},
$$

which holds for all $k \neq m_{j}$. Then, there exists a positivedefinite matrix $\widetilde{P} \geqslant P_{k}$ such that

$$
C_{i}(\widetilde{P}-\bar{P}) C_{i}^{T} \geq \delta_{0}^{i}+\frac{L+1}{L} \sigma_{i} \geq C_{i}\left(P_{k}-\bar{P}\right) C_{i}^{T},
$$

which holds for all $k \neq m_{j}$.

On the other hand, Lemma 1 indicates $\delta_{m_{j}}^{i} \geq 0$. From (47), we have that

$$
C_{i}\left(P_{m_{j}}-\bar{P}\right) C_{i}^{T}-\sigma_{i}>\delta_{m_{j}}^{i} \geq 0,
$$

which holds for all $k=m_{j}$, and from Theorem 1, we have that

$$
P_{m_{j}}<H_{0}\left(P_{m_{j-1}}\right)=A P_{m_{j-1}} A^{T}+Q<A \widetilde{P} A^{T}+Q,
$$

which holds for all $k=m_{j}$.

Combining (50) and (52), we take the positive-definite matrix $S=\max \left\{\widetilde{P}, A \widetilde{P} A^{T}+Q\right\}$, then we have that

$$
P_{k} \prec S=\max \left\{\widetilde{P}, A \widetilde{P} A^{T}+Q\right\},
$$

which holds for all $k \in \mathbb{Z}_{+}$, and Theorem 2 shows that $\bar{P}<P_{k}$ holds for all $k \in \mathbb{Z}_{+}$. Thus, $\bar{P}<P_{k}<S$ holds for all $k \in \mathbb{Z}_{+}$. This completes the proof.

Notice that, in view of Theorems 2 and 3, the DVTSbased Kalman filter initialized at sampling time $k=0$ with a priori initial covariance matrix $P_{0 \mid 0}=\Pi \succ 0$ ensures that the prediction variance matrix $P_{k}$ is positive definite and uniformly bounded from both above and below for any sampling time $k$, which ensures that the estimation error is also uniformly bounded in mean square for any sampling time $k$.

\section{Simulation Results}

In this section, the developed distributed cooperative fusion estimation approach with variance-based triggering scheme is applied to a single-target tracking problem, where the sensors are deployed to track the state of a moving target, whose motion model is given as follows: 


$$
\begin{aligned}
& x(k)=\left(\begin{array}{cccc}
1 & T & 0 & 0 \\
0 & 1 & 0 & 0 \\
0 & 0 & 1 & T \\
0 & 0 & 0 & 1
\end{array}\right) x(k-1)+\omega(k-1), \\
& y_{i}(k)=\left(\begin{array}{cccc}
1 & 0 & 0 & 0 \\
0 & 0 & 1 & 0
\end{array}\right) x(k)+v_{i}(k),
\end{aligned}
$$

where $x(k)=\left[p_{x}(k), v_{x}(k), p_{y}(k), v_{y}(k)\right]^{T}$ denotes the position and velocity components along the $x$-axes and $y$ axes, respectively; $y_{i}(k)$ is the observation of the target's position provided by the $i$-th sensor for $i=1,2, \ldots, N$; $T$ is the sampling time interval; and $\omega(k-1)$ is a zero-mean Gaussian process noise with the covariance

$$
Q=q\left(\begin{array}{cccc}
\frac{T^{3}}{3} & \frac{T^{2}}{2} & 0 & 0 \\
\frac{T^{2}}{2} & T & 0 & 0 \\
0 & 0 & \frac{T^{3}}{3} & \frac{T^{2}}{2} \\
0 & 0 & \frac{T^{2}}{2} & T
\end{array}\right),
$$

and $q>0 ; v_{i}(k)$ is the observation noise assumed be zeromean Gaussian process noise with the covariance $R_{i}=r I_{2 \times 2}$ and $r>0$. To improve the reliability and the overall performance of the estimation systems, a wireless network of five smart sensors is deployed to cooperatively sense the motion state of a moving target, and each smart sensor only transmits or shares its processed measurement to its nearby sensors.

In order to provide a quantitative comparison of what the total triggering number is on each smart sensor, the same problem setting with three different transmission scenarios is considered.

(i) The periodic triggering scheme (PTS): this case can be achieved by setting $\sigma_{i} \equiv 0$ and $\delta_{k}^{i} \equiv 0$ in (12) and (13).

(ii) The static variance-based triggering scheme (SVTS): this case is presented in (16), and the threshold parameters in (16) are selected as $\sigma_{1}=0.015, \sigma_{2}=$ $0.015, \sigma_{3}=0.016, \sigma_{4}=0.019$, and $\sigma_{5}=0.016$.

(iii) The dynamic variance-based triggering scheme (DVTS): the threshold parameters in (12)-(15) are selected as $\sigma_{1}=0.01, \delta_{0}^{1}=0.005(0.01+0.005=0.0$ 15), $\sigma_{2}=0.01, \delta_{0}^{2}=0.005(0.01+0.005=0.015), \sigma_{3}$ $=0.01, \delta_{0}^{3}=0.006(0.01+0.006=0.016), \sigma_{4}=0.01$, $\delta_{0}^{4}=0.009(0.01+0.009=0.019), \sigma_{5}=0.01, \delta_{0}^{5}=0$. $006(0.01+0.006=0.016)$, and $L=5$.
The system and noise parameters in above three scenarios are chosen as $r_{1}=0.1, r_{2}=0.2, r_{3}=0.3, r_{4}=0.4, r_{5}=0.5$, and $q=1.0$.

The simulation results for the above single-target tracking problem are shown in Figures 2-5. To facilitate comparison analysis, we define a trigger ratio (TR) of each smart sensor as a triggering performance index by $J_{\mathrm{tr}}^{i}=N_{\mathrm{atr}}^{i} / N_{\mathrm{ptr}}^{i}$, where $N_{\mathrm{atr}}^{i}$ denotes the actually total triggering number of smart sensor $i$ and $N_{\mathrm{ptr}}^{i}$ denotes the total triggering number of sensor $i$ computed by the PTS. Note that in the PTS case, the total triggering number of each smart sensor is 100 , which means that the TR is $J_{\mathrm{tr}}^{i}=100 \%$ for each sensor. However, in the STVS case, we find that the TR of sensor 1 is $74 \%$, sensor 2 is $74 \%$, sensor 3 is $73 \%$, sensor 4 is $74 \%$, and sensor 5 is $73 \%$. In contrast, implementing the DVST (12)-(15), the TR of each sensor is significantly reduced to $J_{\mathrm{tr}}^{1}=45 \%, J_{\mathrm{tr}}^{2}=48 \%$, $J_{\mathrm{tr}}^{3}=47 \%, J_{\mathrm{tr}}^{4}=49 \%$, and $J_{\mathrm{tr}}^{5}=44 \%$, respectively. A detailed comparison of the triggering number of each sensor in different cases is illustrated in Figure 2. Therefore, we conclude that the DVTS is more efficient in reducing the rate of sampling and communication among sensors than the SVTS and the PTS, thus has more potential to ease the continual occupancy of the limited resources, and Figure 3 shows the specific triggering instants of each sensor node in the SVTS case and the DVTS case. We can see from Figure 3 that the average intertrigger times generated by the DVTS are always no less or larger than the ones generated by the STVS.

To facilitate comparison of the estimation performance between different triggering schemes, the quadratic estimation error (QEE) and average quadratic estimation error (AQEE) are used and presented as follows, respectively:

$$
\begin{aligned}
\operatorname{QEE}(k) & =\frac{1}{L_{M}} \sum_{h=0}^{L_{M}}\left(x(k)-\hat{x}_{i}^{h}(k \mid k)\right)^{T}\left(P_{k / k}^{i}\right)^{h}\left(x(k)-\hat{x}_{i}^{h}(k \mid k)\right), \\
\operatorname{AQEE} & =\frac{1}{S_{t}} \frac{1}{L_{M}} \sum_{k=0}^{S_{t}} \sum_{h=0}^{L_{M}}\left(x(k)-\hat{x}_{i}^{h}(k \mid k)\right)^{T}\left(P_{k / k}^{i}\right)^{h}\left(x(k)-\hat{x}_{i}^{h}(k \mid k)\right),
\end{aligned}
$$

where $L_{M}$ is the total number of tests, $S_{t}$ is the total steps of the estimation, $x(k)$ denotes the actual state of the moving target, and $\hat{x}_{i}^{h}(k \mid k)$ and $\left(P_{k / k}^{i}\right)^{h}$ denote the estimation state and the estimation error covariance of the moving target estimated by the sensor $i$ at the sampling time $k$ in the $h$-th test.

The simulation results between the SVTS case and the DVTS case are analyzed by calculating the QEE and the AQEE on each sensor and are demonstrated in Figures 4 and 5. It is shown in Figures 4 and 5 that the SVTS and the DVTS provide approximately the same QEE, which means that the DVTS does not provide larger QEE than the ones by applying the SVTS even through less total triggering number. In addition, Table 1 shows that the AQEE provided by the DVTS is slightly larger than the one provided by the SVTS, which means that the DVTS with less total triggering number requires a small sacrifice of the AQEE performance. To sum up, the overall superiority of the developed DVTS over the PTS and the SVTS is verified. 


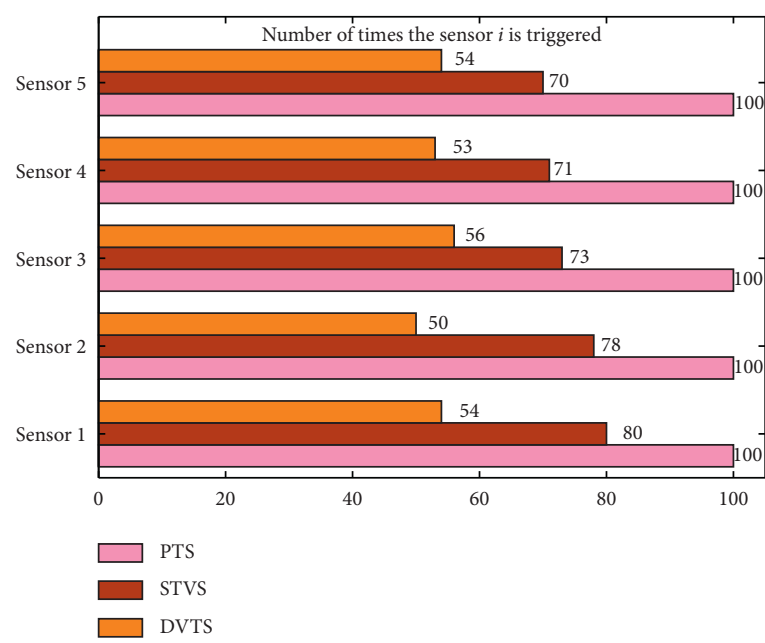

(a)

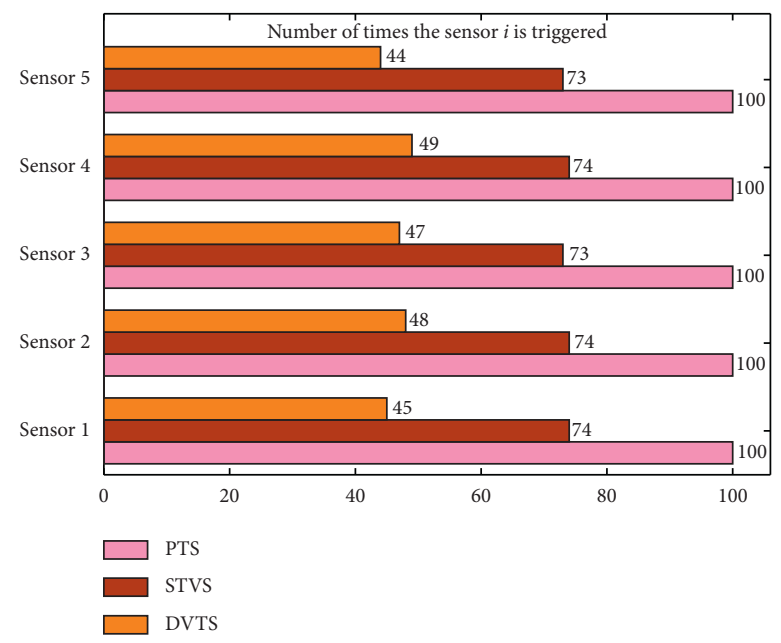

(b)

FIGURE 2: Total triggering number of each smart sensor in different cases of the PTS, the SVTS, and the DVTS: (a) straight trajectory; (b) sinusoidal trajectory.
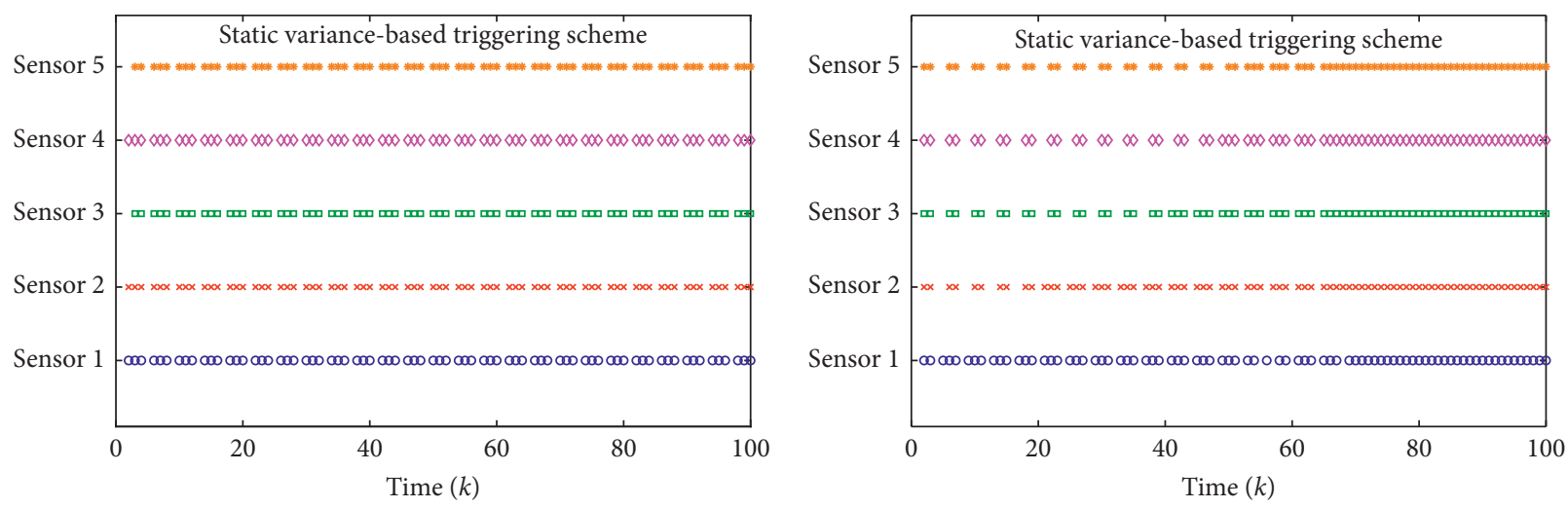

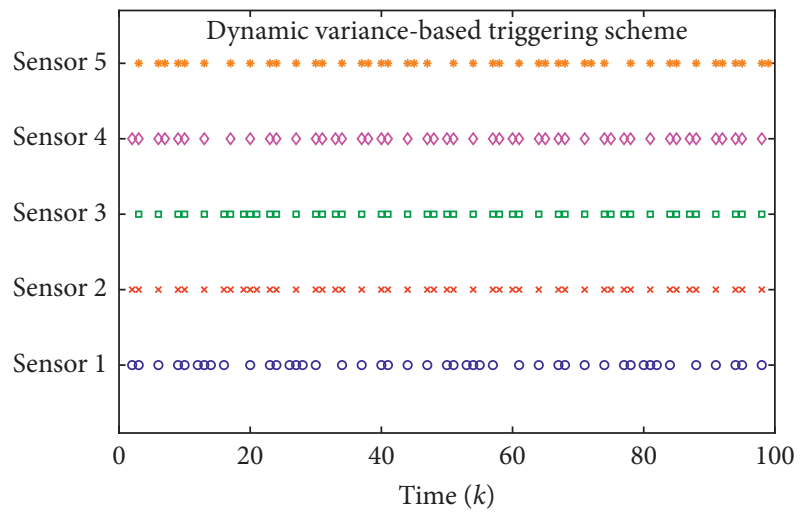

(a)

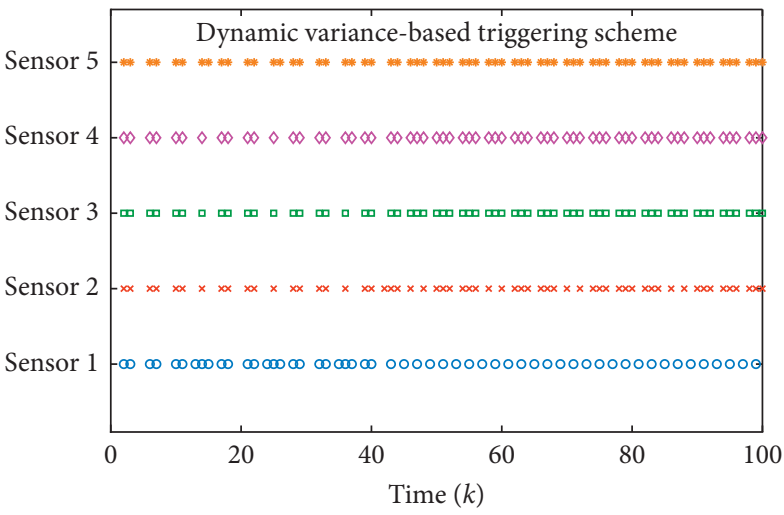

(b)

FIGURE 3: Specific triggering instants of each smart sensor in different cases of the SVTS and the DVTS: (a) straight trajectory; (b) sinusoidal trajectory. 


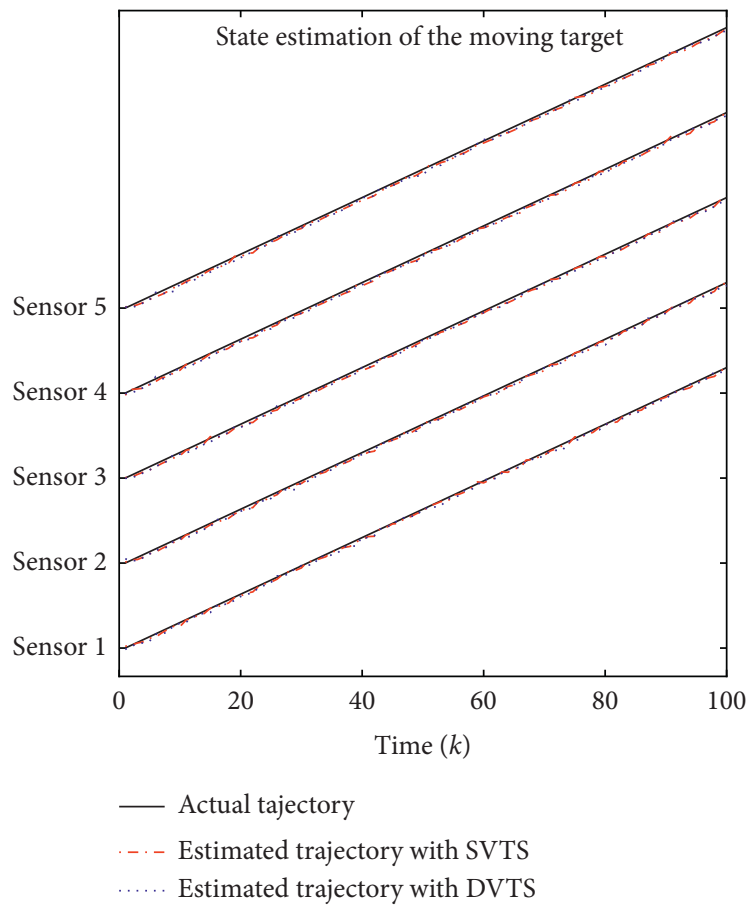

(a)

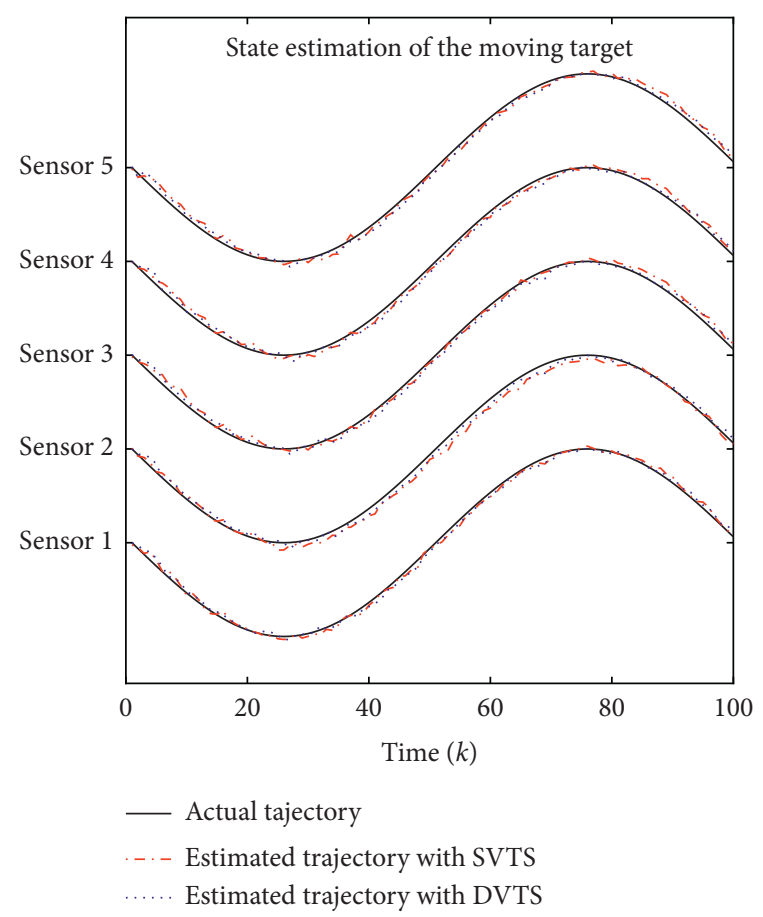

(b)

FIgURE 4: Actual trajectory of the moving target and its estimated trajectories on sensor $i$ in different cases of the SVTS and the DVTS: (a) straight trajectory; (b) sinusoidal trajectory.
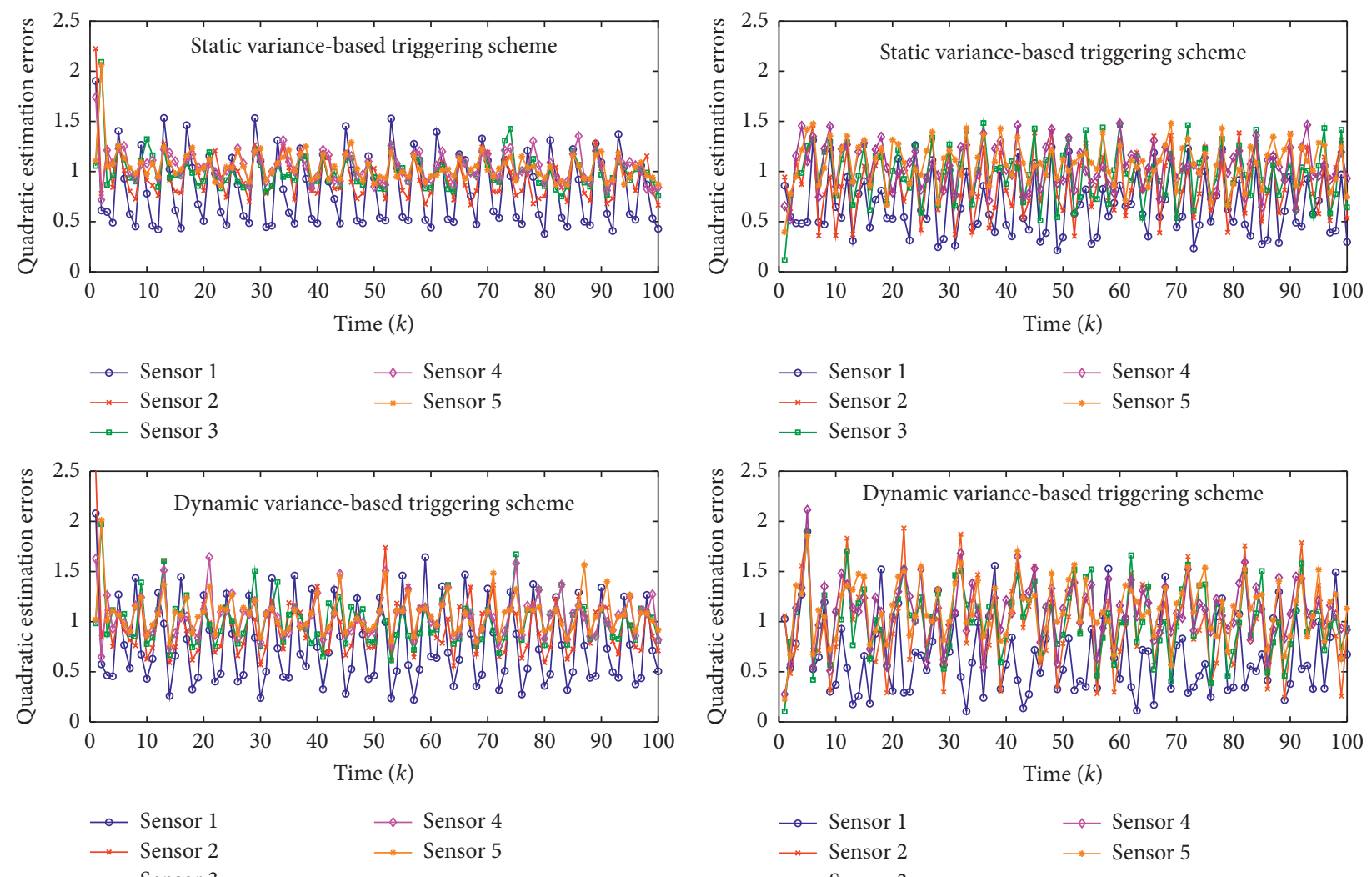

(a)

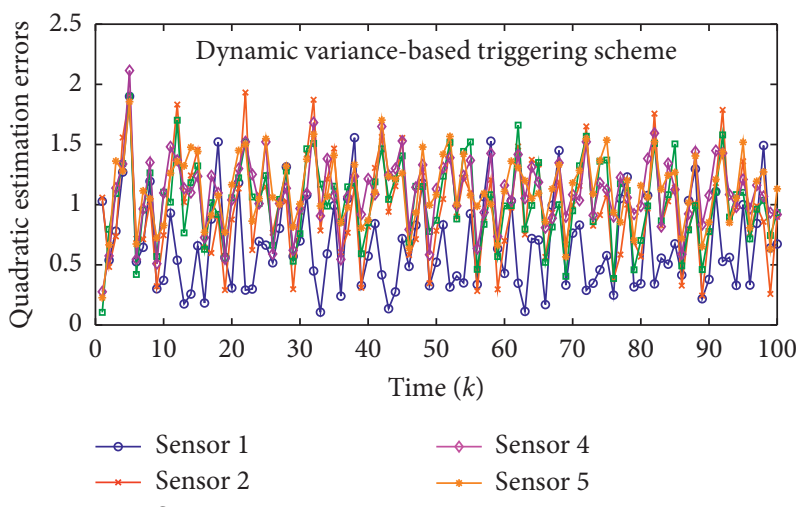

(b)

FIgURE 5: Quadratic estimation error $\varepsilon_{k}^{i}$ of sensor $i$ in different cases of the SVTS and the DVTS: (a) straight trajectory; (b) sinusoidal trajectory. 
TABLE 1: Average quadratic estimation error of sensor $i$ in different cases of the SVTS and the DVTS.

\begin{tabular}{lcccc}
\hline & \multicolumn{2}{c}{ Straight trajectory } & \multicolumn{2}{c}{ Sinusoidal trajectory } \\
& $\begin{array}{c}\text { AQEE- } \\
\text { SVTS }\end{array}$ & $\begin{array}{c}\text { AQEE- } \\
\text { DVTS }\end{array}$ & $\begin{array}{c}\text { AQEE- } \\
\text { SVTS }\end{array}$ & $\begin{array}{c}\text { AQEE- } \\
\text { DVTS }\end{array}$ \\
\hline Sensor 1 & 0.4127 & 0.4360 & 0.6662 & 0.6745 \\
Sensor 2 & 0.6180 & 0.7472 & 0.8747 & 0.9708 \\
Sensor 3 & 0.7098 & 0.8065 & 0.9753 & 1.0189 \\
Sensor 4 & 0.7711 & 0.8494 & 1.0449 & 1.1013 \\
Sensor 5 & 0.8106 & 0.8775 & 1.0787 & 1.1196 \\
Average & 0.6644 & 0.7433 & 0.9280 & 0.9770 \\
\hline
\end{tabular}

\section{Conclusion}

In this paper, the distributed cooperative estimation problem for discrete linear stochastic dynamical systems over resource-constrained wireless sensor networks has been addressed. What time does each smart sensor need to observe or sense the dynamical system and transmit its local measurement to its nearby sensors, it is determined by the DVTS. The distributed cooperative estimators with SVTS and DVTS have been delicately established and a new type of Riccati equation has been derived as a critical element of the SVTS and the DVTS for these estimators. The stability and accuracy of the proposed estimation method is investigated, and the boundedness of the expected estimation error covariance is analyzed. Finally, a moving target tracking model has been employed to illustrate the effectiveness and advantage of the proposed approach, which can be also used in many other applications, especially in the field of the collaborative perception of WSNs, such as cooperative target localization and tracking, cooperative control and guidance of mobile robots, and smart grids.

\section{Data Availability}

The data used to support the findings of this study are available from the corresponding author upon request.

\section{Conflicts of Interest}

The authors declare that there are no conflicts of interest regarding the publication of this paper.

\section{Acknowledgments}

This work was supported in part by the National Nature Science Foundation of China under Grant no. 62003001, in part by Anhui Provincial Natural Science Foundation under Grant no. 2008085QF298, in part by University Natural Science Research Project of Anhui Province (China) under Grant no. KJ2019A0104, in part by Science Research Foundation for Young Teachers of Anhui University of Science and Technology under Grant no. QN2018115, and in part by Preresearch Project of National Natural Science Foundation of Anhui University of Science and Technology under Grant no. YY201801.

\section{References}

[1] A. Ribeiro, I. D. Schizas, S. I. Roumeliotis, and G. Giannakis, "Kalman filtering in wireless sensor networks: reducing communication cost in state-estimation problems," IEEE Control Systems Magazine, vol. 30, pp. 66-86, 2010.

[2] C. Liang, F. Wen, and Z. Wang, "Trust-based distributed kalman filtering for target tracking under malicious cyber attacks," Information Fusion, vol. 46, pp. 44-50, 2019.

[3] W. Lee, M. Bae, H. Kim, and H. Kim, "Mobile device-centric access point monitoring scheme for handover decision triggering in heterogeneous networks," International Journal of Communication Systems, vol. 30, pp. 1-e3376.16, 2017.

[4] A. Ez-Zaidi and S. Rakrak, "A comparative study of target tracking approaches in wireless sensor networks," Journal of Sensors, vol. 2016, Article ID 3270659, 11 pages, 2016.

[5] H. Zhu and M. Luo, "Hybrid robust sequential fusion estimation for wsn-assisted moving-target localization with sensor-node-position uncertainty," IEEE Transactions on Instrumentation and Measurement, vol. 69, no. 9, pp. 64996508, 2020.

[6] H. Fu, Z. Sharif Khodaei, and M. H. F. Aliabadi, "An eventtriggered energy-efficient wireless structural health monitoring system for impact detection in composite airframes," IEEE Internet of Things Journal, vol. 6, no. 1, pp. 1183-1192, 2019.

[7] E. Xu, Z. Ding, and S. Dasgupta, "Target tracking and mobile sensor navigation in wireless sensor networks," IEEE Transactions on Mobile Computing, vol. 12, no. 1, pp. 177-186, 2013.

[8] Y. Zeng, R. Zhang, and T. J. Lim, "Wireless communications with unmanned aerial vehicles: opportunities and challenges," IEEE Communications Magazine, vol. 54, no. 5, pp. 36-42, 2016.

[9] E. Fadel, V. C. Gungor, L. Nassef et al., "A survey on wireless sensor networks for smart grid," Computer Communications, vol. 71, pp. 22-33, 2015.

[10] W.-A. Zhang, X. Yang, L. Yu, and S. Liu, "Sequential fusion estimation for rss-based mobile robots localization with event-driven wsns," IEEE Transactions on Industrial Informatics, vol. 12, no. 4, pp. 1519-1528, 2016.

[11] J. H. Yoo, W. Kim, and H. J. Kim, "Event-driven Gaussian process for object localization in wireless sensor networks," in Proceedings of the 2011 IEEE/RSJ International Conference on Intelligent Robots and Systems, pp. 2790-2795, San Francisco, CA, USA, September 2011.

[12] S. Trimpe and R. D'Andrea, "Event-based state estimation with variance-based triggering," IEEE Transactions on Automatic Control, vol. 59, no. 12, pp. 3266-3281, 2014.

[13] J. P. Hespanha, P. Naghshtabrizi, and Y. Xu, "A survey of recent results in networked control systems," Proceedings of the IEEE, vol. 95, no. 1, pp. 138-162, 2007.

[14] G. Battistelli, A. Benavoli, and L. Chisci, "Data-driven communication for state estimation with sensor networks," Automatica, vol. 48, no. 5, pp. 926-935, 2012.

[15] X. Liu and A. Goldsmith, "Kalman filtering with partial observation losses," in Proceedings of the 2004 43rd IEEE Conference on Decision and Control (CDC)(IEEE Cat. No. 04CH37601), Nassau, Bahamas, December 2004.

[16] B. Sinopoli, L. Schenato, M. Franceschetti, K. Poolla, M. I. Jordan, and S. S. Sastry, "Kalman filtering with intermittent observations," IEEE Transactions on Automatic Control, vol. 49, no. 9, pp. 1453-1464, 2004.

[17] Y. Mo, R. Ambrosino, and B. Sinopoli, "Sensor selection strategies for state estimation in energy constrained wireless 
sensor networks," Automatica, vol. 47, no. 7, pp. 1330-1338, 2011.

[18] J. Wu, Q.-S. Jia, K. H. Johansson, and L. Shi, "Event-based sensor data scheduling: trade-off between communication rate and estimation quality," IEEE Transactions on Automatic Control, vol. 58, no. 4, pp. 1041-1046, 2013.

[19] D. Han, Y. Mo, J. Wu, S. Weerakkody, B. Sinopoli, and L. Shi, "Stochastic event-triggered sensor schedule for remote state estimation," IEEE Transactions on Automatic Control, vol. 60, no. 10, pp. 2661-2675, 2015.

[20] M. Lemmon, "Event-triggered feedback in control, estimation, and optimization," in Networked Control Systems, pp. 293-358, Springer, Berlin, Germany, 2010.

[21] Q. Tan, X. Dong, Q. Li, and Z. Ren, "Distributed eventtriggered cubature information filtering based on weighted average consensus," IEET Control Theory \& Applications, vol. 12, pp. 78-86, 2017.

[22] H. Liu and H. Yu, "An event-triggered approach to robust state estimation for wireless sensor networks," Journal of Control and Decision, vol. 4, no. 4, pp. 263-275, 2017.

[23] D. Shi, T. Chen, and L. Shi, "Event-triggered maximum likelihood state estimation," Automatica, vol. 50, no. 1, pp. 247-254, 2014.

[24] D. Shi, T. Chen, and L. Shi, "An event-triggered approach to state estimation with multiple point- and set-valued measurements," Automatica, vol. 50, no. 6, pp. 1641-1648, 2014.

[25] K. You, N. Xiao, and L. Xie, "Kalman filtering with scheduled measurements," in Analysis and Design of Networked Control Systems, pp. 269-291, Springer, Berlin, Germany, 2015.

[26] D. Han, Y. Mo, J. Wu, and L. Shi, “An opportunistic sensor scheduling solution to remote state estimation over multiple channels," IEEE Transactions on Signal Processing, vol. 64, no. 18, pp. 4905-4917, 2016.

[27] D. Han, K. You, L. Xie, J. Wu, and L. Shi, "Optimal parameter estimation under controlled communication over sensor networks," IEEE Transactions on Signal Processing, vol. 63, no. 24, pp. 6473-6485, 2015.

[28] S. Weerakkody, Y. Mo, B. Sinopoli, D. Han, and L. Shi, "Multi-sensor scheduling for state estimation with eventbased, stochastic triggers," IEEE Transactions on Automatic Control, vol. 61, no. 9, pp. 2695-2701, 2016.

[29] L. Li, D. Yu, Y. Xia, and H. Yang, "Remote nonlinear state estimation with stochastic event-triggered sensor schedule," IEEE Transactions on Cybernetics, vol. 49, pp. 1-12, 2018.

[30] Z. Jin, Y. Hu, and C. Sun, "Event-triggered state estimation for stochastic hybrid systems with missing measurements," IET Control Theory \& Applications, vol. 12, no. 18, pp. 2551-2561, 2018.

[31] H. Sandberg, M. Rabi, M. Skoglund, and K. H. Johansson, "Estimation over heterogeneous sensor networks," in Proceedings of the 2008 47th IEEE Conference on Decision and Control, Cancun, Mexico, December 2008.

[32] X. Ge, Q.-L. Han, and Z. Wang, "A dynamic event-triggered transmission scheme for distributed set-membership estimation over wireless sensor networks," IEEE Transactions on Cybernetics, vol. 49, no. 1, pp. 171-183, 2019.

[33] B. D. Anderson and J. B. Moore, Optimal Filtering, Courier Corporation, Chelmsford, MA, USA, 2012. 